$\begin{array}{ll}\text { Research Square } & \begin{array}{l}\text { Preprints are preliminary reports that have not undergone peer review. } \\ \text { They should not be considered conclusive, used to inform clinical practice, } \\ \text { or referenced by the media as validated information. }\end{array}\end{array}$

\title{
Circular RNA circMYL1 inhibit proliferation and promote differentiation of myoblasts by sponging miR-2400
}

Ibrahim E. Elnour

Northwest A\&F University https://orcid.org/0000-0003-3663-7129

Xiaogang Wang

Northwest A\&F University

Toremurat Zhansaya

Northwest A\&F University

Akhatayeva Zhanerke

Northwest A\&F university

Rajwali Khan

Northwest A\&F University

Jie Cheng

Northwest A\&F University

Yongzhen Huang

Northwest A\&F University

Xianyong Lan

Northwest A\&F University

Chuzhao Lei

Northwest A\&F University

Hong Chen ( $\square$ chenhong1212@263.net)

College of Animal Science and Technology

Research

Keywords: circMYL1, miR2400, bovine primary myoblasts, proliferation, differentiation

Posted Date: August 17th, 2020

DOI: https://doi.org/10.21203/rs.3.rs-49630/v1

License: (c) (1) This work is licensed under a Creative Commons Attribution 4.0 International License. Read Full License

Version of Record: A version of this preprint was published at Cells on January 16th, 2021. See the published version at https://doi.org/10.3390/cells10010176. 


\section{Abstract \\ Background}

Circular RNAs (circRNAs) are a class of endogenous non-coding RNAs (ncRNAs) involved in regulating skeletal muscle development by sponging microRNAs (miRNAs). This study aimed to define the circMYL1 molecular mechanisms in the regulation of bovine myogenesis and to disclose its regulatory mechanism through miR-2400 interaction.

\section{Methods}

The potential role of circMYL1 was identified in the proliferation of bovine myoblast through mRNA and protein expression of proliferation marker genes (PCNA, CyclinD1, and CDK2), cell counting kit-8 (CCK8) assay, flow cytometry analysis, and EdU assay. Analysis of the expression of differentiation marker genes ( $M y o D, M y o G$, and $M Y H 2$ ) and immunofluorescence of Myosin heavy chain $(M y H C)$ was used to assess cell differentiation.

\section{Results}

The proliferation analysis revealed that circMYL 1 inhibited the proliferation of bovine primary myoblast. Furthermore, the differentiation analysis demonstrated that circMYL1 promoted the differentiation of bovine primary myoblast. The luciferase screening and RNA immunoprecipitation (RIP) assays found that circMYL 1 could have interaction with miR-2400. Additionally, we demonstrated that miR-2400 promoted proliferation and inhibited differentiation of bovine primary myoblast, while circMYL 1 may eliminate the effects of miR-2400, as showed by rescue experiments.

\section{Conclusions}

Our results revealed that a novel circular RNA of circMYL 1 could inhibit proliferation and promote differentiation of myoblast by sponging miR-2400.

\section{Introduction}

Mammals have three different types of muscle tissues, including cardiac muscle, skeletal muscle, and smooth muscle[1]. Skeletal muscle represents half of the total body mass and has a critical role for voluntary motion and support[1]. The vertebrate skeletal muscle originates from the somites during embryonic development[2]. Previous studies showed that myogenesis is a complicated and orchestrated process, regulated by several genes, signaling pathways, and network regulation[3, 4]. For instance, the multiple specifics myogenic regulatory transcription factors (MRFs) that are important for the regulation of the skeletal muscle development. Previous studies found that MRFs are a member of basics helix-loop-helix (bHLH), including MYOG, MyoD, Myf5, and MRF4. MRFs that significantly expressed in skeletal muscle suggested that they have an essential role during the proliferation and differentiation of skeletal muscle[5]. In addition, non-coding RNAs such as microRNAs (miRNAs), long non-coding RNAs (Linc RNAs) and circular RNAs (circRNAs) have been reported to be involved in the development of skeletal muscle and play critical roles during myogenesis[6-8]. Nevertheless, to explore the molecular regulatory mechanisms during muscle development and meat quality need further in-depth experiments [9].

Circular RNAs (circRNAs) are a class of endogenous non-coding RNAs (ncRNAs) that have a covalently closed circular structure with no 5 ' end cap and 3 ' end poly(A) tail, more stable compared with linear RNA molecules, produced by back-splicing from precursor mRNA[10, 11]. CircRNAs have high expression in the cytoplasm of eukaryotic cells[12,13], and can be circRNAs can exist for a long time in various cell and tissue at specific development stages[14]. Recent studies divided the circRNAs into three groups; 1 ) exon-derived circRNAs (exonic circRNAs), which are formed by exon splicing of the coding gene, including one or Multiple exon sequences $[15,16]$; 2) Intron circRNA (circular intronic RNA, ciRNA), which without of exon sequences and produced by cyclization of the coding gene intron sequences [17]; 3) exon-intron circRNA (ElciRNA), which is a fusion circRNA that contains both an exon and an intron sequence[18, 19]. Furthermore, it is found that circRNAs are involved in the transcription and post-transcriptional regulation of gene through different modes of action [18]. Previous studies noted that most circRNAs contain miRNA binding sites. Therefore, circRNA can act as a highly efficient competitive endogenous RNA (ceRNA), effectively sponging miRNAs and regulating miRNA target genes[20, 21]. For instance, circRNAs, ciRS-7, and Sry inhibit the function of miRNA through sponging miR-7 and miR-138, respectively[13, 22]. Besides sponging miRNAs, circular RNA may also bind to RNA-binding proteins (RBPs) to form complexes of RNA proteins (RPCs) [23]. In addition, it was stated that (ElciRNAs) interact with small nuclear ribonucleoproteins of RNA polymerase II and U1 and cis-induce host-gene transcript in the nucleus[24]. CirRNAs have been reported to play critical roles during skeletal muscle proliferation and differentiation. For instance, it was noted that the circFGFR4 overexpression promotes myogenic differentiation of bovine primary myoblasts by sponging miR-107 [25]. Furthermore, it was also investigated that the circZfp609 sponges miR-194-5p to inhibit myogenic differentiation in C2C12 [26]. Another research also found that circ $L M O 7$ overexpression promotes proliferation and inhibits bovine primary myoblasts differentiation by sponging miR-378a-3p [27]. Similarly, CircFUT10 inhibits proliferation and promotes differentiation of bovine primary myoblasts by sponging miR-133a [28], whereas the overexpression of circSNX29 promotes myoblasts differentiation and inhibits myoblast proliferation by sponging miR-744 and activate the $\mathrm{Wnt}^{2} \mathrm{a} / \mathrm{Ca}^{2+}$ signaling pathway [29]. We stated in our previous research that circ MYL 1 expressed differently between adult and fetal of bovine muscle, suggesting that circ $M Y L 1$ may affect the development of muscles [27].

MicroRNAs (miRNAs) are about 17 to 24 nucleotides in a class of small single-stranded endogenous, non-coding RNA. Usually, they bind to specific target mRNAs (3-UTR) to induce mRNA degradation or translation inhibition[30]. Several studies reported that miRNA involved in multiple physiological and biochemical processes, such as carcinogenesis, hematopoiesis, neurogenesis, cell proliferation, and cell apoptosis[31, 32]. Glazov et al. reported that miRLoading [MathJax]/jax/output/CommonHTML/fonts/TeX/fontdata.js 
2400 is not recognizable in other mammalian genomes. It is, therefore, a specific bovine miRNA [33]. Recently, it was reported that the overexpression of miR2400 promotes the proliferation of skeletal muscle-derived satellite cells (MDSCs) by targeting the MYOG gene [34]. However, the function of miR-2400 in the regulation of myoblast differentiation was not reported.

This study aimed to define the circMYL1 molecular mechanisms in the regulation of bovine myogenesis and to disclose its regulatory mechanism through miR-2400 interaction. We found that circMYL1 inhibits proliferation and promotes differentiation of bovine primary myoblasts by acting as a sponge of miR2400 and activated MYOG gene. The findings may be fruitful to understand the role of circMYL 1 in myogenesis and will provide a platform for the study of myogenesis based on circular RNA experiments.

\section{Materials And Methods Tissue samples collection}

In this study, the animal samples have been approved by the Northwest Agriculture and Forestry University's Animal Care and Use Committee. At a local slaughterhouse in (Xi'an; China), all specimens from Qinchuan bovine embryonic phase ( 90 days) were collected, including Skeletal muscle, stomach, intestine, spleen, heart, liver, lung, and kidney, and washed by (DEPC) water, and stored at $-80^{\circ} \mathrm{C}$ until RNA extraction.

\section{RNA extraction and Real-time PCR}

Total cell or tissue RNAs were extracted using the manufacturer's instructions for TRIzol Reagent (Takara, Dalian, China). Next, using the PrimerScript RT reagent kit with gDNA Eraser (Takara, Dalian, China), RNA was reversed to complementary DNA (cDNA). And a nucleoplasmic separation kit (PARIS kit, Life Technologies, Carlsbad, CA, USA) was used to separate the nuclear and cytoplasmic fractions. For treatment with RNase R, $1 \mu \mathrm{g}$ of total RNA was incubated at $37^{\circ} \mathrm{C}$ for 20 mints with two units $\mu \mathrm{g}^{-1}$ of RNase R and then purified using an RNeasy MinElute cleaning kit (Qiagen, Hilden, Germany). SYBR Green PCR master mix reagent kit (Takara, Dalian, China) was used to conduct qRT-PCR. The GAPDH and U6 (for miRNA) were used as an internal control for data normalization with three biological replicates. The relative expression level of mRNA was calculated using the $2^{-\Delta \Delta C t}$ technique. All primers used in this study are listed in Table 1.

\section{Plasmid Construction}

To explore the possible functions of circMYL 1 during bovine skeletal muscle development, we downloaded the sequencing data of circRNAs of bovine muscle tissue from NCBI: accession ID,GSE87908;databankURL,https://www.ncbi.nlm.nih.gov/geo/query/acc.cgi?token=atglausktpsjlel\&acc=GSE87908 (Text S1). For the construction of circMYL1 overexpression plasmids, using PCR to amplify the linear sequences of circ $M Y L 1$, and the $C D N A$ template was produced from the bovine primary myoblasts RNA by RT-PCR. The obtained linear sequences were then cloned into the $\mathrm{Kpnl}$ and $\mathrm{BamHI}$-restriction sites of the pCD2.1 vector (Invitrogen, Carlsbad, CA, USA) according to the manufacturer's instruction, to produce the overexpression vector PCD2.1- circMYL1. The MYOG 3'UTRWT fragment, including the miR-2400 binding site, was amplified and inserted into the psiCHECK-2 vector (MYOG-WT) (Promega, Madison, WI, USA) at the 3 ' end of the Renilla gene using the Xhol and Not/ restriction enzymes (Takara, Dalian, China) and T4 DNA ligase. Mutant type psiCHECK-2- MYOG 3'UTR-MUT (MYOG-MUT) was created using overlapping PCR to mutate complementary to the miR-2400 seed region. MYOG-WT and MYOG-MUT are eventually incorporated in the same way into the psiCHECK-2 vector. A miR-2400 sensor (with a perfect miR-2400 binding site) was created by inserting two sequences that complement the mature miR-2400 sequence after the Rluc psiCHECK-2 vector. Consequently, the fluorescence activity alteration will react to miR-2400 reflecting. The PCK-circMYL1 vector was also obtained using the same procedure. Primer sequences are shown in Table 2 . All constructs were verified by sequencing (Sangon Biotech, Shanghai, China).

\section{Cells culture}

The bovine primary myoblasts, as reported previously [35], were isolated and cultured from bovine longissimus muscle. The HEK293T cells (ATCC, USA) and bovine primary myoblasts were grown in growth medium containing high-glucose Dulbecco's modified Eagle's medium (DMEM; Hyclone, USA) and supplemented by $10 \%$ or $20 \%$ fetal bovine serum (FBS) (Hyclone, USA) and $1 \%$ penicillin-streptomycin (Invitrogen, USA) and incubated at $37{ }^{\circ} \mathrm{C}$ with $5 \%$ CO 2 . The differentiation of bovine primary myoblast was induced by replacing growth medium with the differentiation medium (DMEM containing $2 \%$ horse serum) when bovine primary myoblasts confluence reached approximately $70 \% \sim 80 \%$.

\section{Cells transfection}

The bovine primary myoblasts were cultured in six-well plates. When the confluence of the cells reached to $40 \%$ for proliferation or $70 \% \sim 80 \%$ for differentiation the cells were transfected with PCD2.1-circMYL1 $(2 \mu \mathrm{g} / \mathrm{ml}), 100 \mathrm{nM}$ si-circMYL1 (RiboBio, Guangzhou, China), $50 \mathrm{nM}$ miR-2400 mimics (RiboBio, Guangzhou, China) using Lipofectamine 2000 (Invitrogen, USA) according to the manufacturer's protocol. Transcription was inhibited by adding $1 \mathrm{mM}$ of Actinomycin D solution to the cell culture medium (Leagene, Beijing, China).

\section{RNA Fluorescent In Situ Hybridization (RNA-FISH)}

Specific probes to circMYL1 sequence were used to perform in situ hybridization in bovine primary myoblasts following instructions from the probe manufacturer (RiboBio, Guangzhou, China). The probe sequence is 5'- GGCATTCATCTCTTCATAGTTGATGCA - 3'. The cells were cultured in the 6-well plates in cover glass and grown to a confluence of $70 \% \sim 80 \%$, and then fixed. After treatment with $0.1 \%$ of Triton X- 100 transmembrane, cells were incubated overnight at $37^{\circ} \mathrm{C}$ with $20 \mathrm{mg} / \mathrm{ml}$ of circMYL1 probe. Then using DAPI to counterstain the nuclei. Photos have been obtained using a confocal laser microscope FV1200 (Olympus)

\section{5-Ethvnvl 2'-Deoxvuridine Assav (EdU)}

Loading [MathJax]/jax/output/CommonHTML/fonts/TeX/fontdata.js 
Cell-Light EdU Apollo ${ }^{\circledR}$ (RiboBio; China) was used to detect the proliferation of bovine primary myoblasts. The experiment was carried out following the protocols of the manufacturer. The bovine primary myoblast cells were grown in a 96-well plate and then transfected by treatments with three replicates in each group. Concisely, before immunostaining, the cells were incubated at $37^{\circ} \mathrm{C}$ with $50 \mu \mathrm{M}$ EdU for 2 hours after 24 hours of transfection. A fluorescent microscope (AMG EVOS, Seattle, WA) was used to capture all images.

\section{Cell Counting Kit-8 (CCK8) assay}

The cell counting reagent Kit-8(CCK8) (Multiscience, Hangzhou, China) was used to assess the proliferation of cells. The bovine primary myoblasts were grown in 96 well plates with eight independent replicates for each treatment. After 24 hours of cultured at $37^{\circ} \mathrm{C}$, we added $10 \mu \mathrm{L}$ of $\mathrm{CCK}-8$ reagent to each well for 4 hours. Then an automatic microplate reader (Molecular Devices, USA) was used to measure the absorbance value of all samples at $450 \mathrm{~nm}$.

\section{Cell Cycle Assay by Flow Cytometry}

Bovine primary myoblasts were seeded in $60 \mathrm{~mm}$ cell culture plates $\left(2 \times 10^{6}\right.$ cells/well). After 24 hours of transfected, we collected cells and washed with PBS buffer, and resuspended with $1 \mathrm{ml}$ of DNA staining solution and $10 \mu \mathrm{L}$ of permeabilization solution (Multisciences, Hangzhou, China). The suspension was then incubated for 30 minutes in the dark at room temperature. Flow Cytometry (FACS Canto TM II, BD Biosciences, USA) measured the cell cycle and, for each treatment group, there were three independent replicates.

\section{Luciferase Activity Assay}

For investigated the binding sites of circMYL1 with miR-2400, HEK293T cells were seeded in 96-well plates. Then, the miR-2400 mimics and PCK-circMYL1 or miR-2400 sensors and PCD2.1-circMYL 1 are cotransfected with Lipofectamine 2000 into HEK293 T cells. The miR-2400 mimics and MYOG-WT or MYOG-MUT have also been cotransfected into cells. After 24 hours, the cells were then washed by Phosphate Buffered Saline (PBS) and collected using $100 \mu \mathrm{L}$ Passive Lysis Buffer (PLB). Luciferase activity assay was performed using MPPC luminescence analyzer (HAMAMATSU, Beijing, China), and Dual-Luciferase ${ }^{8}$ Reporter (DLR) Assay Kit (Promega, Madison, WI, USA). And the activities of Firefly luciferase were normalized in each well to Renilla luminescence.

\section{Western Blotting Analysis}

Total bovine primary myoblasts proteins were extracted from different treatment groups using RIPA lysis buffer containing 1 mM PMSF (Solarbio; Beijing, China). The protein extract was then boiled for 10 minutes in a loading buffer of $5 \times$ SDS-PAGE. The protein was then separated by SDS-PAGE and transferred to a $0.2 \mu \mathrm{m}$ polyvinylidene fluoride membranes (PVDF). Then incubated for 2 hours at room temperature with $5 \%$ skim milk in Tris Buffered Saline, with Tween (TBST) to block the PVDF membrane. Subsequently, in $4^{\circ} \mathrm{C}$ overnight incubation with the primary anti-MYOD specific antibodies (Dilution 1:1000; ab16148; Abcam, England), anti-MYOG (1;1000; ab124800; Abcam, Cambridge, England), anti-MYH2 (Dilution 1:1000; WL02785; Wanleibio, China), anti- CDK2 (Dilution 1:1000; WL02028; Wanleibio, China), anti-PCNA (Dilution 1:500; WL03069; Wanleibio, China), anti-CyclinD1 (Dilution 1:1000; WL01435a; Wanleibio, China), anti- $\beta$-actin (1:1000; KM9001T, SungeneBiotech, China). After that, the PVDF membranes were washed with TBST buffer and then incubated at room temperature for 2 hours with the secondary antibody. The secondary antibodies were: goat anti-mouse IgG HRP (M21001S, Abmart, China), goat anti-rabbit IgG (H+L)-HRP (BA1054, Boster, China). Finally, ECL luminous liquid (DiNing, China) was used to detect the protein bands.

\section{Immunofluorescent Staining}

Primary bovine myoblasts seeded in 24-well plates and differentiation was induced to form the myotubes for four days. The differentiated cells were fixed for 30 minutes with $4 \%$ paraformaldehyde, then washed with PBS for 5 minutes three times. The cells were subsequently permeabilized by adding (Triton X-100) $0.5 \%$ for 15 minutes. The cells were then incubated overnight with the MyHC-antibody (1:250; GTX20015, GeneTex, USA), which was diluted in 1\% Bovine Serum Albumin. The cells were washed with 1XPBS and incubated with IgG H\&L secondary anti-mouse (Alexa Fluor 594) (1:500; RS3608, Immunoway, USA) and protected for 2 hours from light at room temperature. The nuclei of cells were stained for 15 minutes with DAPI (4',6-diamidino-2-phenylindole) (Solarbio; Beijing, China). A fluorescence microscope was used to capture the Immunofluorescence images (DM5000B, Leica, Germany).

\section{Statistical Analysis}

The data of the experiment are presented as mean \pm SEM. SPSS 20.0 software was used for statistical analysis, and the student t-test was used to determine the variation between the groups. Variations in $* P<0.05$ and $* * P<0.01$ were considered statistically significant.

\section{Results}

\section{Characterization of circMYL1}

To confirm circMYL 1's circular nature, we designed two pairs of primers in a divergent and convergent direction (Fig. 1A), the cDNA or genomic DNA (gDNA) was used as amplification templates. As shown in (Fig. 1B), the divergent primers produced only in cDNA samples a single distinct band; however, the convergent primers amplified the products from both the cDNA and the gDNA samples, which indicates that these circRNAs are bovine genome back-splicing products. The PCR products were sequenced using Sanger sequencing for the verification of the circMYL 1 junction site (Fig. 1C), which was consistent with the sequencing results. The circularity of circMYL1 was confirmed by RNase R. Total RNAs were treated with RNase R exonuclease, and qRT-PCR was performed, the results showed that the circMYL1 was more resistant to RNase R than MYL 1 mRNA (Figs. 1D and 1E). Furthermore, we investigated the stability and location of circMYL1 in bovine primary myoblast. After treatment with Actinomycin $\mathrm{D}$, a transcription inhibitor, total RNA was collected at the specified time points. Then, we evaluated the expression level of circMYL1 and MYL 1 mRNA. The results showed that the circMYL 1 was highly stable with a half-life of transcript exceeding 24 hours, while the corresponding linear transcript was the half-life of 4 hours (Fig. 1F). In order to investigate the cellular localization of circMYL1, we conducted semi-quantitative PCR analysis of nuclear and cytoplasmic circMYL 1 and RNA fluorescent in situ hybridization (FISH) Loading [MathJax]/jax/output/CommonHTML/fonts/TeX/fontdata.js ack-splicing region to determine its subcellular location. Results showed that circMYL 1 was located 
mainly in the cytoplasm and also was presented in the nucleus, indicating that circ $M Y L 1$ could regulate gene expression at the post-transcription level and may regulate of their parental genes (Figs. $1 \mathrm{G}$ and $1 \mathrm{H}$ )[36, 37]. Moreover, we detected the expression level of circMYL 1 in different developmental tissues using quantitative real-time polymerase chain reaction (qRT-PCR), to inspect the potential role of circMYL1 in growth and development, particularly in bovine muscle development (Fig. 11). The samples, including heart, liver, muscle, kidney, lung, spleen, stomach, and intestine, were collected from the fetal of cattle. The qRT-PCR analysis found that the circMYL 1 showed a high expression level in some tissues, including muscle, liver, stomach, and heart, compared with intestine, kidney, lung, and spleen in the fetal stage. The circ $M Y L 1$ showed considerable expression pattern in muscle tissues, suggesting that circ $M Y L 1$ plays an essential role in myogenesis. Furthermore, we detected the expression level of circ $M Y L 1$ during bovine primary myoblast proliferation and differentiation. We found that the expression level of circMYL 1 gradually decreased during proliferation and increased from $0 \mathrm{~d}$ to $5 \mathrm{~d}$ during differentiation (Fig. $1 \mathrm{~J}$ ). Taken together, our findings indicated that circMYL1 could be a positive regulator and stable circRNA for the development of muscles.

\section{circMYL1inhibits myoblasts proliferation}

In order to explore the function of circMYL 1 in the proliferation of skeletal muscle cells, we conducted overexpression and knocked down experiments by transfecting circMYL 1overexpression vector and small interfering RNAs (siRNAs) that was designed to target circMYL 1 back-splicing (pCD2.1-circMYL1 and si-circMYL 1) to bovine primary myoblast. The relative expression of circMYL 1 was detected by qRT-PCR after $48 \mathrm{~h}$ post-transfection, resulting in both the effect of overexpression and knockdown, reaching a substantial level (Fig. 2A-3A). Furthermore, we detected the proliferation process of bovine primary myoblast by flow cytometry for cell cycle analysis, cell counting kit-8 (CCK-8), 5-ethynyl-2'-deoxyuridine (EdU) incorporation assays, qRT-PCR, and western blotting assays after transfecting with pCD2.1-circMYL1/NTC, or si-circMYL1/si-NC. Firstly, we detected the effect of circMYL1on expression of the proliferation marker genes ( $P C N A, C y C l i n D 1$, and $C D K 2)$ using qRT-PCR and western blotting and the results showed that the overexpression of circMYL1 significantly decreased the expression of these genes at the mRNA and protein levels (Fig. $2 \mathrm{~B}$ and $2 \mathrm{C}$ and $2 \mathrm{C}^{\prime}$ ). Then, the cell cycle analysis revealed that overexpression of circMYL1 decreased the number of cells in the $S$ phase and increased the number of cells in the G0/G1 phase (Fig. 2D and 2D'). The results further confirmed by using EdU staining. The results showed that the number of EdU-positive cells was less than that in the control group (Fig. $2 \mathrm{E}$ and $2 \mathrm{E}$ '). Furthermore, the CCK-8 assay revealed that circMYL 1 possessed lower proliferation vitality than the negative control (Fig. $2 \mathrm{~F}$ ). In addition, we found that the knockdown of the circMYL1 increased the expression of the proliferation marker genes (PCNA, CyclinD1, and CDK2) at the mRNA and protein levels (Fig. 3B and $3 C$ and $\left.3 C^{\prime}\right)$. The cell cycle analysis revealed that si- circ $M Y L 1$ increased the number of cells in the $S$ phase and decreased the number of cells in $\mathrm{G} 0 / \mathrm{G} 1$ (Fig. 3D and 3D'). Moreover, knockdown of circ MYL1 markedly increased the number of EdU labelled cells compared with the negative control group (Fig. 3E and $\left.3 E^{\prime}\right)$. Furthermore, the cell counting assay (CCK8) demonstrated that transfected with si-circMYL1 possessed higher proliferation vitality than the negative control (Fig. 3F). These results revealed that circMYL1 inhibits the proliferation of bovine primary myoblasts.

\section{CircMYL1 promotes myoblasts differentiation}

To investigate the possible roles of circMYL1 in the regulation of skeletal muscle differentiation, the bovine primary myoblasts were transfected by pCD2.1circMYL 1/NTC or si-circMYL1/si-NC and differentiated cells for four days. Then, the cells were harvested, and qRT-PCR and western blotting analysis were conducted to detect the expression level of the differentiation marker gene (MyoD, MyoG, and $M Y H 2)$. The result of qRT-PCR demonstrated that the overexpression of circMYL1 increased the expression levels of $M y O D, M y o G$, and $M Y H 2$ compared with the control group (Fig. 4A). In contrast, the knockdown of circMYL 1 decreased the expression level of $M y O D, M y o G$, and $M Y H 2$ at mRNA compared with the si-NC group (Fig. 4D). Similarly, the western blotting analysis showed that the overexpression of circMYL 1 increased the protein levels of MyoD, MyoG, and MYH2 compared with the control group (Fig. $4 \mathrm{~B}$ and $\left.4 B^{\prime}\right)$, whereas, the knockdown of circMYL1 decreased the protein level of MyoD, MyoG, and MYH2 compared with the si-NC group (Fig. 4E and 4E'). Collectively, these results indicated that circ $M Y L 1$ may promote bovine myoblasts differentiation. Furthermore, the results from the immunofluorescence experiment found that circ MYL 1 overexpression enhanced the formation of myotube (Fig. 4C), whereas the knockdown of circMYL 1 reduced the formation of myotube (Fig. 4F).

\section{CircMYL1 Acts as a Sponge for miR-2400}

Previously, it has found that circular RNA act as miRNA sponge and circMYL 1 could inhibit proliferation and promote differentiation of bovine primary myoblasts. We conjectured that circMYL Texerts function by sponging miRNA and regulating miRNA expression. To screen the potential of miRNAs binding with circMYL1, the RNAhybrid assay was used to conduct the putative complementarity site between circMYL1 and miRNA. Remarkably, we found that circMYL 1 has potential miR-2400 binding site (Fig. 5A). The dual luciferase assay was used to explore whether circMYL 1 target miR-2400 or not. The result revealed that miR-2400 reduced the Rluc expression of psiCHECK-2-circMYL1 (PCK-circMYL1) in HEK293T cells (Fig. 5B). Furthermore, we generated a sensor for miR-2400 by inserting two copies of the miR-2400 complementary sequence into the psiCHECK-2 vector (Fig. 5C). The result showed that miR-2400 markedly reduced the Rluc activity of the miR-2400 sensor in HEK293T cells, on the other hand, the overexpression of the circMYL1 partially returned the decreased Rluc activity that made by binding miR-2400 in a dose-dependent manner (Fig. 5D). Furthermore, RNA immunoprecipitation (RIP) assay was conducted in bovine primary myoblasts to confirm the interaction between circMYL 1 and miR-2400, and qRT-PCR results showed positive enrichment of miR2400 (Fig. 5E) and circMYL1 (Fig. 5F) in Argonaute 2 (Ago2) pull-down samples as compared to negative control (IgG), indicating that circMYL 1 binds to miR2400 through the Ago2 protein. Moreover, the transfection with circMYL1 inhibits the relative expression level of miR-2400 in bovine primary myoblasts (Fig. 5G). Additionally, we analyzed the expression level of miR-2400 during myogenesis in bovine primary myoblasts, and the result showed that the expression of miR-2400 was increased during the proliferation period and decreased during differentiation (Fig. $5 \mathrm{H}$ ). From these results, the expression levels of miR-2400 were opposite to circ $M Y L 1$ expression during myogenesis. Altogether, these finding indicated that circ $M Y L 1$ regulate proliferation and differentiation of bovine primary myoblast by sponging miR-2400.

\section{miR-2400 promotes myoblasts proliferation}

To explore the role of miR-2400 during myoblast proliferation, the bovine primary myoblasts at $40 \%$ density were transfected, by $50 \mathrm{nM}$ miR-2400 mimic and $50 \mathrm{nM}$ mimic necative control and nCD2 1-circMYI 1/NTC for $48 \mathrm{~h}$. The expression of miR-2400 was increased by mimic compared with mimic control Loading [MathJax]/jax/output/CommonHTML/fonts/TeX/fontdata.js 
(Fig. 6A). Various methods, such as qRT-PCR, western blotting, cell counting kit-8 assay, and EdU, have been used to detect the potential role of miR-2400 in the proliferation of myoblast. The qRT-PCR results demonstrated that the transfected by miR-2400 mimic increased the relative expression levels of proliferation marker genes ( $P C N A, C y c l i n D 1$, and $C D K 2)$ at mRNA level, but this result was abolished when transfected by overexpression of circMYL 1(Fig. 6B). Similarly, the western blotting analysis showed that the miR-2400 overexpression increased the protein expression level of (PCNA, CyclinD1, and CDK2) genes, and also, this result was reversed by transfected by circMYL1 overexpression (Fig. $6 \mathrm{C}$ and $6 \mathrm{C}^{\prime}$ ). Analysis of the cell cycle showed that the miR-2400 mimic increased the number of cells in the S phase and decreased the proportion of cells in the G0/G1 phase, indicating that miR-2400 may promote the proliferation of bovine myoblast (Fig. 6D and 6D'). Moreover, 5-ethynyl-2'-deoxyuridine (EdU) analysis and cell counting assay (CCK-8) and showed that overexpression of miR-2400 significantly up-regulated the cell proliferation (Fig. $6 \mathrm{E}$ and $6 \mathrm{E}^{\prime}$ and $6 \mathrm{~F}$ ). However, the result showed that when we co-transfected the miR-2400 mimic and PCD2.1-circMYL 1 into bovine primary myoblasts, the overexpression of circMYL 1 eliminated the effect of miR-2400 on the proliferation of myoblasts (Fig. 6D -6F).

\section{miR-2400 inhibits myoblasts differentiation}

Potential roles of miR-2400 in the regulation of skeletal muscle differentiation were analyzed through transfecting bovine primary myoblasts with miR-2400 mimic or PCD2.1-circMYL 1 at $70 \%-80 \%$ density and differentiated cells for four days. The cells were collected, qRT-PCR and western blotting analysis were conducted for the expression analysis of the differentiation marker genes (MyOD, MyoG, and MYH2). The result of qRT-PCR demonstrated that the miR-2400 mimic decreased the expression levels of $M y O D, M y O G$, and $M Y H 2$ at mRNA compared with the control group, in contrast, when co-transfected with PCD2.1circMYL 1 and miR-2400, the expression levels of $M y O D, M y O G$, and $M Y H 2$ at mRNA were increased (Fig. 7A). Furthermore, the western blotting analysis showed that the miR-2400 mimic decreased the protein levels of MyoD, MyoG, and MYH2 compared with the control group, whereas, co-transfected with PCD2.1-circMYL1 and miR-2400, increased the protein level of MyoD, MyoG, and MYH2 (Fig. 7B and 7B'). Immunofluorescence assay showed that miR-2400 inhibited $M y H C$ expression and the formation of myotube, but circMYL 1 overexpression reversed this effect to some extent (Fig. 7D). Collectively, these results confirmed that miR-2400 inhibits the differentiation of bovine primary myoblasts; however, these effects can be eliminated by overexpression of circMYL 1 . In addition, these findings also showed that circMYL 1 promotes myogenesis by sponging miR-2400.

\section{CircMYL1 acts as a ceRNA for miR-2400 to activate MYOG}

Online bioinformatics suit TargetScan 7.2 was used to clarify miR-2400's possible molecular regulatory mechanism for inhibiting proliferation and promoting differentiation of bovine primary myoblasts. MYOG was considered as a potential target gene for miR-2400 (Fig. 8A). We then constructed MYOG-3'-UTR (wild [WT] and mutant [MUT]) luciferase reporter vectors that contain potential miR-2400 binding sites (Fig. 8B). The two plasmids were transfected separately into HEK293T cells with miR-2400 mimic or negative control. MYOG-WT's luciferase activity was significantly $(P<0.05)$ decreased by miR-2400 but did not affect MYOG-MUT (Fig. 8C). To confirm the involvement of circMYL 1 in the miR-2400 mediated the regulation of MyoG expression, bovine primary myoblast was transfected by pCD2.1-circMYL1 and/or miR-2400 mimic, and the result showed that the overexpression of miR-2400 decreased MyoG expression at mRNA and protein level (Fig. 8D and 8E and 8E'); however, this effect was reversed when transfected with circMYL1. All these results, suggesting that circMYL1 regulates the proliferation and differentiation of bovine myoblasts by acting as a miR-2400 sponge to activate the MYOG gene (Fig. 9).

\section{Discussion}

Recently, a large number of circular RNAs have been identified in different cells and tissues as the results of the development of high-throughput sequencing[15,38]. CirRNAs play a significant biological role in many physiological and pathological processes as a new post-transcription regulator and are a hot topic in biological research[39]. However, the regulatory role of circRNAs in the growth and development of skeletal muscle is still unclear. Myosin light chain 1 (MYL1) is a well-known gene, particularly related to calcium regulation, and plays a vital role in muscle growth and differentiation[40]. It was found that $M Y L 1$ is a critical gene for skeletal muscle function and the deficiency of $M Y L 1$ related to the severe congenital myopathy[41]. The circMYL 1 derived from exon 4 and 5 of the MYL 1 gene. CircRNAs are more stable and longer half-life compared to linear mRNAs because of its covalently closed circular structure [14]. Recently, several studies have proved the crucial roles of circular RNAs in the proliferation and differentiation of myoblast. There confirmed the role of circFUT10 [28], circSVIL [8], circFGFR4 [25], circFGFR2 [42], and circSNX29 [29] in the enhance of myogenesis through positively regulated myoblast differentiation. Moreover, it was found that circ LMO7 [27], circZNF609 [26], negatively regulated the myoblast differentiation.

In our previous publication, the whole-genome sequencing data explored highest expression level of circMYL1 (FPKM = 1869.8) between differentially expressed circular RNAs (up-regulated), and differentially expressed between adult and fetal of bovine muscle tissues suggesting that circ $M Y L 1$ have an essential role in muscle development[27]. In the current study, we investigated the possible role of circMYL 1 in muscle development. The expression of circMYL 1 in different cattle tissues was highly expressed in skeletal muscle. Additionally, the expression decreased during the proliferation and increased during primary myoblast differentiation. These results suggesting that the circMYL 1 may inhibit proliferation promote differentiation of skeletal muscle. In our previous research, we confirmed that circFUT10 and circSNX29 inhibit proliferation and promote differentiation of bovine primary myoblasts [28, 29]. To assess this hypothesis, firstly, we studied the function of circ $M Y L 1$ in bovine primary myoblast proliferation. The results showed that the mRNA and protein expression levels of proliferation marker genes such as PCNA, CyclinD1, and CDK2 were decreased in circMYL1-overexpressed cells, whereas the opposite effects were detected in circMYL1-knockdown cells. Moreover, the EdU incorporation analysis exposed that EdU-positive cells were reduced by overexpression of circMYL 1 and increased by knockdown of circMYL1. Furthermore, the cell counting assay (CCK8) and cell cycle analysis showed that circMYL 1 reduced the proliferation of myoblasts. Based on these results, we strongly postulated that circ $M Y L 1$ has a vital role in myoblast cell proliferation. Furthermore, we also found that the overexpression of circMYL1 increased the expression levels of differentiation marker genes (MyoD, MyoG, and $M Y H 2)$; however, the expression levels decreased by knockdown of circMYL1. The protein levels of MyoD, MyoG, and MYH2 showed similar expression. Myosin heavy chain immunofluorescence also confirmed that circ $M Y L 1$ promoted the differentiation of bovine primary myoblasts into myotubes. These results further confirming the vital function of circMYL 1 in the skeletal muscle cell, and it can promote myoblast differentiation.

Loading [MathJax]/jax/output/CommonHTML/fonts/TeX/fontdata.js 
Previously, it was investigated that circRNAs can act as competing for endogenous RNAs, which can sponge miRNAs to regulate the expression of posttranscription genes. In our previous studies we reported that some circRNAs regulated the development of bovine skeletal muscles by sponging various miRNAs, circ TTN acts as a miR-432 sponge to promote myoblast proliferation and differentiation through the /GF2/PI3K/AKT signaling pathway [43],circSNX29 inhibit cell proliferation and promote cell differentiation by sponging miR-744 to activate the Wnt5a/ca ${ }^{2+}$ pathway[29], circ LMO7 sponge miR$378 a-3 p$ to inhibit cell differentiation and promote cell proliferation of myoblast [27], and circFGFR4 promote cell differentiation by sponging miR-107 to increase the expression of Wnt3a[25]. In this research, we used the bioinformatics software to predict the potential targeting miRNAs, RNAhybrid assay showed that circMYL 1 have possible biding site for miR-2400. Furthermore, we validated that the miR-2400 as targeting for circ $M Y L 1$ using the dual-luciferase reporter assay, RNA immunoprecipitation, qRT-PCR, and western blotting analysis. The results suggested that circMYL1 inhibits proliferation and promotes differentiation of myoblast by sponging miR-2400. It was reported that miR-2400 is a novel and specific bovine miRNA because it is not detectable in other mammalian genomes [33]. It was reported that in skeletal muscle-derived satellite cells (MDSCs), the overexpression of miR-2400 promotes proliferation by targeting the MYOG gene[34]. In this study, we also find the same function of miR-2400 in bovine primary myoblast proliferation. Furthermore, we evaluated the vital role of miR-2400 in myoblast differentiation; miR-2400 inhibits the bovine primary myoblast differentiation, which was never done before. The findings were suggesting that miR-2400 acts as a negative regulatory factor during myoblasts differentiation. Conversely, during muscle development, the roles of miR-2400 were opposite to the effects of circMYL1. Therefore, circ MYL 1 may act as a molecular sponge for miR-2400. Furthermore, we found that circMYL 1 inhibited the stimulatory effects of miR-2400 on the proliferation and eliminated the inhibitory effects of miR-2400 in the differentiation of bovine primary myoblast.

\section{Conclusion}

In conclusion, we identified the novel circular RNA of circMYL1, produced by the MYL 1 gene that could regulate the proliferation and differentiation of bovine myoblasts by acting as a miR-2400 sponge to activate MYOG gene. The findings discover a new mechanism involved in muscle development and could be helpful for cattle breeding programs.

\section{Abbreviations}

\section{circRNAs}

Circular RNAs

ncRNAs

Non-coding RNAs

miRNAs

MicroRNAs

Linc RNAs

long non-coding RNAs

CCK8

Cell counting kit-8

EdU

5-Ethynyl 2'-Deoxyuridine

MyHC

Myosin heavy chain

MYL1

myosin light chain 1

\section{MRFs}

myogenic regulatory transcription factors

\section{bHLH}

basics helix-loop-helix

\section{qRT-PCR}

Quantitative Real-Time PCR

RIP

RNA immunoprecipitation

RNA-FISH

RNA Fluorescent In Situ Hybridization

PBS

Phosphate Buffered Saline

PLB

Passive Lysis Buffer

PVDF

polyvinylidene fluoride membranes

TBST

Tris Buffered Saline, with Tween 


\section{Declarations}

\section{Availability of data and materials}

The data are available from the corresponding author on reasonable request.

\section{Acknowledgements}

We would like to thank Northwest A\&F University for support during the study.

\section{Funding}

This work was supported by the National Natural Science Foundation of China『No.31772574), the Program of National Beef Cattle and Yak Industrial Technology System (CARS-37).

\section{Authors' contributions}

I.E.E. carried out all the experiments, analyzed the data and wrote the paper. X.W., T.Z., A.Z., and J. C. participated in partial experiments. R. K., and revised the manuscript. Y. H., X. L., and C. L. participated in the analyzed data, manuscript revision. H. C. designed the experiments, supervised, and participated in the manuscript revision.

\section{Ethics declarations}

\section{Ethics approval}

The animal samples have been approved by the Northwest Agriculture and Forestry University's Animal Care and Use Committee.

\section{Consent for publication}

Not applicable.

\section{Competing interests}

The authors declare that there is no competing of interest.

\section{References}

1. Le Grand F, Rudnicki MA. Skeletal muscle satellite cells and adult myogenesis. Curr Opin Cell Biol. 2007;19(6):628-33.

2. Buckingham M. Gene regulatory networks and cell lineages that underlie the formation of skeletal muscle. Proc Natl Acad Sci USA. 2017;114(23):5830-7.

3. Buckingham M. Myogenic progenitor cells and skeletal myogenesis in vertebrates. Curr Opin Genet Dev. 2006;16(5):525-32.

4. Bismuth K, Relaix F. Genetic regulation of skeletal muscle development. Experimental cell research. 2010;316(18):3081-6.

5. Bentzinger CF, Wang YX, Rudnicki MA. Building muscle: molecular regulation of myogenesis. Cold Spring Harbor perspectives in biology 2012, 4(2).

6. Sun Y, Ge Y, Drnevich J, Zhao Y, Band M, Chen J. Mammalian target of rapamycin regulates miRNA-1 and follistatin in skeletal myogenesis. J Cell Biol. 2010;189(7):1157-69.

7. Legnini I, Morlando M, Mangiavacchi A, Fatica A, Bozzoni I. A feedforward regulatory loop between HuR and the long noncoding RNA linc-MD1 controls early phases of myogenesis. Molecular cell. 2014;53(3):506-14.

8. Ouyang H, Chen X, Li W, Li Z, Nie Q, Zhang X. Circular RNA circSVIL Promotes Myoblast Proliferation and Differentiation by Sponging miR-203 in Chicken. Frontiers in genetics. 2018;9:172.

9. Te Pas MF, Keuning E, Hulsegge B, Hoving-Bolink AH, Evans G, Mulder HA. Longissimus muscle transcriptome profiles related to carcass and meat quality traits in fresh meat Pietrain carcasses. Journal of animal science. 2010;88(12):4044-55.

10. Suzuki H, Tsukahara T. A view of pre-mRNA splicing from RNase R resistant RNAs. Int J Mol Sci. 2014;15(6):9331-42.

11. Rong D, Sun H, Li Z, Liu S, Dong C, Fu K, Tang W, Cao H. An emerging function of circRNA-miRNAs-mRNA axis in human diseases. Oncotarget. 2017;8(42):73271-81.

12. Li Y, Zheng Q, Bao C, Li S, Guo W, Zhao J, Chen D, Gu J, He X, Huang S. Circular RNA is enriched and stable in exosomes: a promising biomarker for cancer diagnosis. Cell research. 2015;25(8):981-4.

13. Memczak S, Jens M, Elefsinioti A, Torti F, Krueger J, Rybak A, Maier L, Mackowiak SD, Gregersen LH, Munschauer M, et al. Circular RNAs are a large class of animal RNAs with regulatory potency. Nature. 2013;495(7441):333-8.

14. Jeck WR, Sorrentino JA, Wang K, Slevin MK, Burd CE, Liu J, Marzluff WF, Sharpless NE. Circular RNAs are abundant, conserved, and associated with ALU repeats. RNA. 2013;19(2):141-57.

15. Salzman J, Gawad C, Wang PL, Lacayo N, Brown PO. Circular RNAs are the predominant transcript isoform from hundreds of human genes in diverse cell types. PloS one. 2012;7(2):e30733.

16. Ashwal-Fluss R, Meyer M, Pamudurti NR, Ivanov A, Bartok O, Hanan M, Evantal N, Memczak S, Rajewsky N, Kadener S. circRNA biogenesis competes with Loading [MathJax]/jax/output/CommonHTML/fonts/TeX/fontdata.js 
17. Zhang Y, Zhang XO, Chen T, Xiang JF, Yin QF, Xing YH, Zhu S, Yang L, Chen LL. Circular intronic long noncoding RNAs. Molecular cell. 2013;51(6):792806.

18. Li Z, Huang C, Bao C, Chen L, Lin M, Wang X, Zhong G, Yu B, Hu W, Dai L, et al. Exon-intron circular RNAs regulate transcription in the nucleus. Nat Struct Mol Biol. 2015;22(3):256-64.

19. Yang $Y$, Fan $X$, Mao M, Song X, Wu P, Zhang Y, Jin Y, Yang Y, Chen LL, Wang Y, et al. Extensive translation of circular RNAs driven by N(6)-methyladenosine. Cell research. 2017;27(5):626-41.

20. Zhong Y, Du Y, Yang X, Mo Y, Fan C, Xiong F, Ren D, Ye X, Li C, Wang Y, et al. Circular RNAs function as ceRNAs to regulate and control human cancer progression. Mol Cancer. 2018;17(1):79.

21. Kim HH, Kuwano Y, Srikantan S, Lee EK, Martindale JL, Gorospe M. HuR recruits let-7/RISC to repress c-Myc expression. Genes Dev. 2009;23(15):1743-8.

22. Hansen TB, Kjems J, Damgaard CK. Circular RNA and miR-7 in cancer. Cancer research. 2013;73(18):5609-12.

23. Tay Y, Rinn J, Pandolfi PP. The multilayered complexity of ceRNA crosstalk and competition. Nature. 2014;505(7483):344-52.

24. Hentze MW, Preiss T. Circular RNAs: splicing's enigma variations. EMBO J. 2013;32(7):923-5.

25. Li H, Wei X, Yang J, Dong D, Hao D, Huang Y, Lan X, Plath M, Lei C, Ma Y, et al: circFGFR4 Promotes Differentiation of Myoblasts via Binding miR-107 to Relieve Its Inhibition of Wnt3a. Molecular therapy Nucleic acids 2018, 11:272-283.

26. Wang Y, Li M, Wang Y, Liu J, Zhang M, Fang X, Chen H, Zhang C. A Zfp609 circular RNA regulates myoblast differentiation by sponging miR-194-5p. Int J Biol Macromol. 2019;121:1308-13.

27. Wei X, Li H, Yang J, Hao D, Dong D, Huang Y, Lan X, Plath M, Lei C, Lin F, et al: Circular RNA profiling reveals an abundant circLMO7 that regulates myoblasts differentiation and survival by sponging miR-378a-3p. Cell death \& disease 2017, 8(10):e3153.

28. Li H, Yang J, Wei X, Song C, Dong D, Huang Y, Lan X, Plath M, Lei C, Ma Y, et al: CircFUT10 reduces proliferation and facilitates differentiation of myoblasts by sponging miR-133a. Journal of cellular physiology 2018, 233(6):4643-4651.

29. Peng S, Song C, Li H, Cao X, Ma Y, Wang X, Huang Y, Lan X, Lei C, Chaogetu B, et al. Circular RNA SNX29 Sponges miR-744 to Regulate Proliferation and Differentiation of Myoblasts by Activating the Wnt5a/Ca(2+) Signaling Pathway. Molecular therapy Nucleic acids. 2019;16:481-93.

30. Bartel DP. MicroRNAs: genomics, biogenesis, mechanism, and function. Cell. 2004;116(2):281-97.

31. Kloosterman WP, Plasterk RH. The diverse functions of microRNAs in animal development and disease. Developmental cell. 2006;11(4):441-50.

32. Liu K, Liu Y, Mo W, Qiu R, Wang X, Wu JY, He R: MiR-124 regulates early neurogenesis in the optic vesicle and forebrain, targeting NeuroD1. Nucleic acids research 2011, 39(7):2869-2879.

33. Glazov EA, Kongsuwan K, Assavalapsakul W, Horwood PF, Mitter N, Mahony TJ. Repertoire of bovine miRNA and miRNA-like small regulatory RNAs expressed upon viral infection. PloS one. 2009;4(7):e6349.

34. Zhang WW, Tong HL, Sun XF, Hu Q, Yang Y, Li SF, Yan YQ, Li GP. Identification of miR-2400 gene as a novel regulator in skeletal muscle satellite cells proliferation by targeting MYOG gene. Biochem Biophys Res Commun. 2015;463(4):624-31.

35. Sun X, Li M, Sun Y, Cai H, Lan X, Huang Y, Bai Y, Qi X, Chen H: The developmental transcriptome sequencing of bovine skeletal muscle reveals a long noncoding RNA, IncMD, promotes muscle differentiation by sponging miR-125b. Biochimica et biophysica acta 2016, 1863(11):2835-2845.

36. Huang C, Shan G. What happens at or after transcription: Insights into circRNA biogenesis and function. Transcription. 2015;6(4):61-4.

37. Chen N, Zhao G, Yan X, Lv Z, Yin H, Zhang S, Song W, Li X, Li L, Du Z. A novel FLI1 exonic circular RNA promotes metastasis in breast cancer by coordinately regulating TET1 and DNMT1. Genome biology. 2018;19(1):218.

38. Veno MT, Hansen TB, Veno ST, Clausen BH, Grebing M, Finsen B, Holm IE, Kjems J. Spatio-temporal regulation of circular RNA expression during porcine embryonic brain development. Genome biology. 2015;16:245.

39. Greco S, Cardinali B, Falcone G, Martelli F. Circular RNAs in Muscle Function and Disease. International journal of molecular sciences 2018, 19(11).

40. Park JW, Lee JH, Kim SW, Han JS, Kang KS, Kim SJ, Park TS. Muscle differentiation induced up-regulation of calcium-related gene expression in quail myoblasts. Asian-Australasian journal of animal sciences. 2018;31(9):1507-15.

41. Ravenscroft G, Zaharieva IT, Bortolotti CA, Lambrughi M, Pignataro M, Borsari M, Sewry CA, Phadke R, Haliloglu G, Ong R, et al. Bi-allelic mutations in MYL1 cause a severe congenital myopathy. Human molecular genetics. 2018;27(24):4263-72.

42. Chen X, Ouyang H, Wang Z, Chen B, Nie Q. A Novel Circular RNA Generated by FGFR2 Gene Promotes Myoblast Proliferation and Differentiation by Sponging miR-133a-5p and miR-29b-1-5p. Cells 2018, 7(11).

43. Wang X, Cao X, Dong D, Shen X, Cheng J, Jiang R, Yang Z, Peng S, Huang Y, Lan X, et al. Circular RNA TTN Acts As a miR-432 Sponge to Facilitate Proliferation and Differentiation of Myoblasts via the IGF2/PI3K/AKT Signaling Pathway. Molecular therapy Nucleic acids. 2019;18:966-80.

\section{Figures}



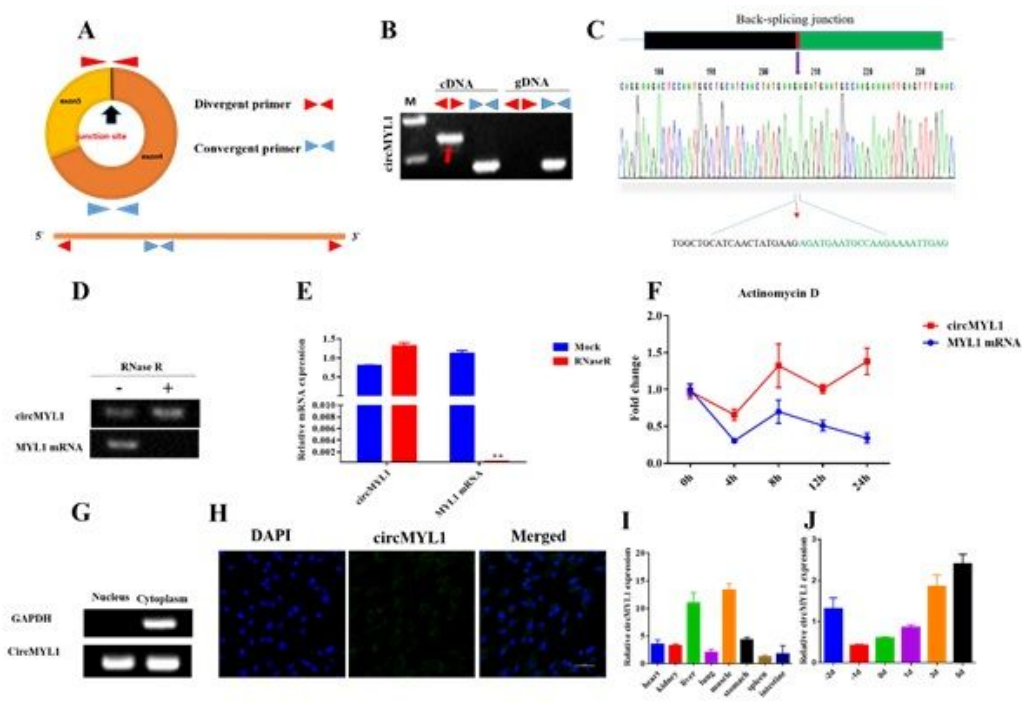

\section{Figure 1}

Characterization of circMYL1 (A) Schematic diagram showing the design of the circMYL1 primers. (B) The divergent primers and convergent primers in cDNA and gDNA samples. (C) Sanger sequencing confirmed the circMYL1 sequence of the back-splicing junction. (D) RNase R verified the existence of circMYL1. (E) qRT-PCR demonstrated the relative expression of circMYL1 and MYL1 mRNA in myoblasts treated with RNase R (F) qRT-PCR detected the relative expression of circMYL1and MYL1 mRNA in bovine primary myoblasts treated with Actinomycin D at the specific time points. (G) The cytoplasm and nuclear expression of circMYL1 were measured by semiquantitative PCR. (H) RNA fluorescence in situ hybridization was performed to detect the subcellular location of circMYL1. Blue shows nuclei stained with DAPI, green shows the RNA probe detecting circMYL1. Scale bar, $50 \mu m$. (I) The expression level of circMYL1 in different fetal tissues. $(\mathrm{J})$ CircMYL1 expression level during proliferation and differentiation of bovine primary myoblasts. Data are shown as mean $\pm \mathrm{SEM}$. $\mathrm{N}=3$.

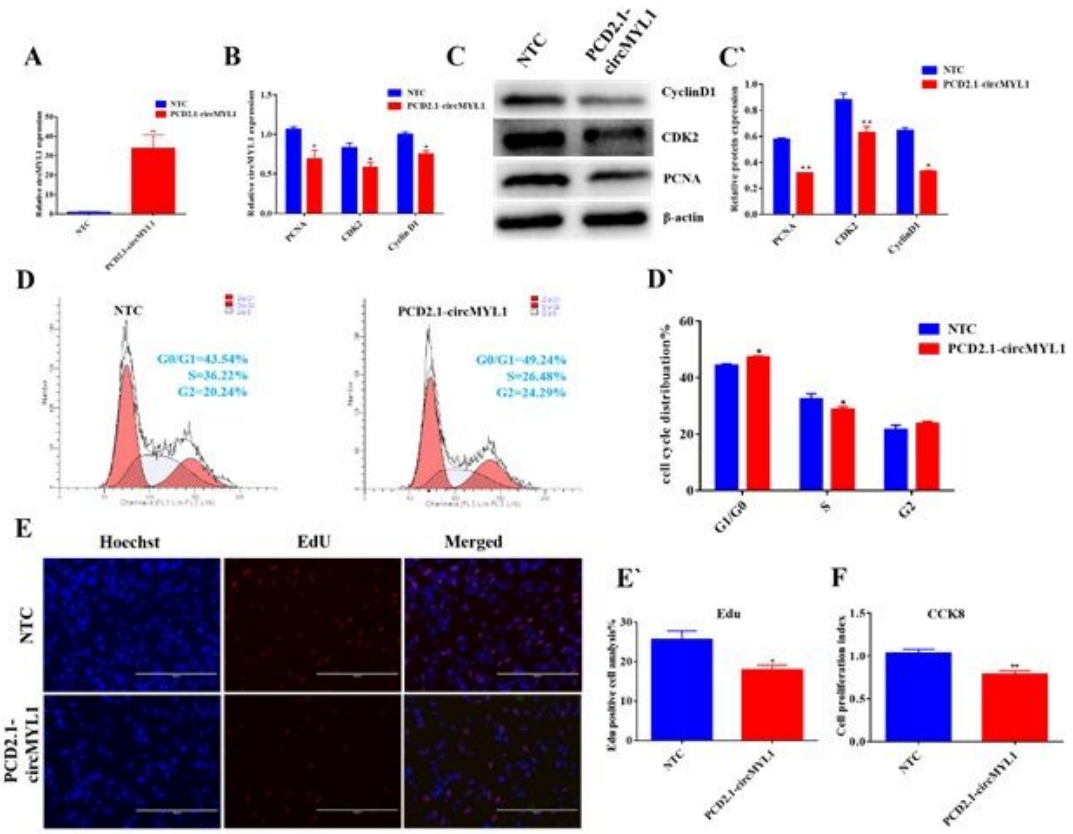

Figure 2

The overexpression of circMYL1 inhibits the proliferation of bovine primary myoblasts (A) qRT-PCR detected the efficiency of the circMYL1 overexpression vector. (B) qRT-PCR detected the relative expression levels of proliferation marker genes PCNA, CDK2, and CyclinD1. (C) western blot detected the protein expression levels of proliferation marker gene PCNA, CDK2, and CyclinD1. (C

) His $\rightarrow$ gramgraphshow $\in$ gtheprote $\in b$ and densityevaluatedusingIma $\geq J$. (D) Thecellcyc $\leq$ wasanalyzedbyflowcy $\rightarrow$ metry. ( $D$ ) Histogram graph showing the number of cells in each phase of the cell cycle. (E) The cell proliferation was detected by (EdU) assay Scale bar, $200 \mu \mathrm{m}$. (E') Graph of a histogram showing the percentage of EdU-positive cells. (F) The cell counting kit-8 (CCK-8) assay was used to detect the cell proliferation index. Data are shown as mean \pm SEM. $N=3 .{ }^{*} P<0.05 ; * * P<0.01$. 

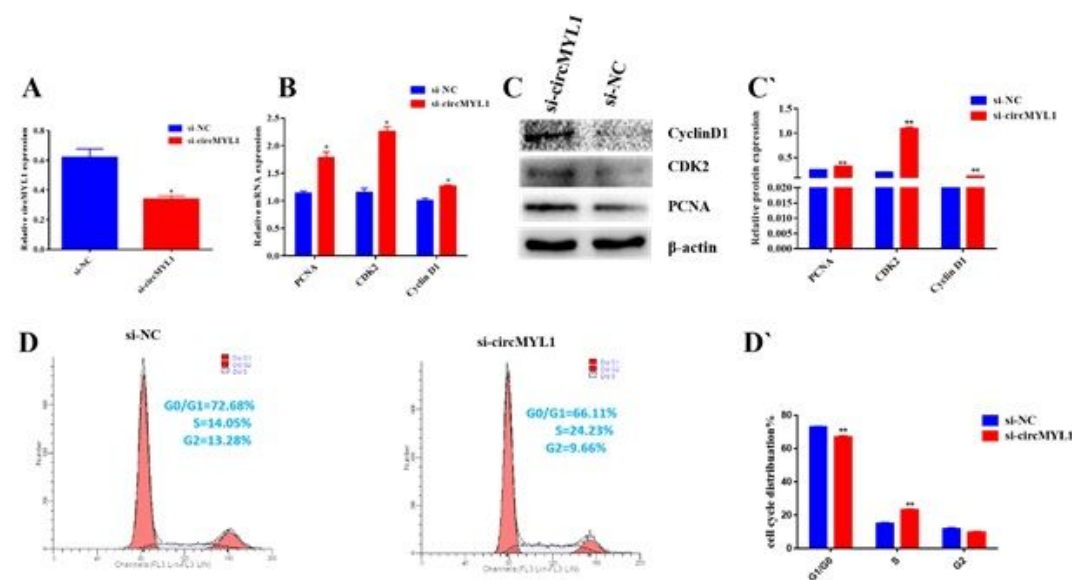

D'
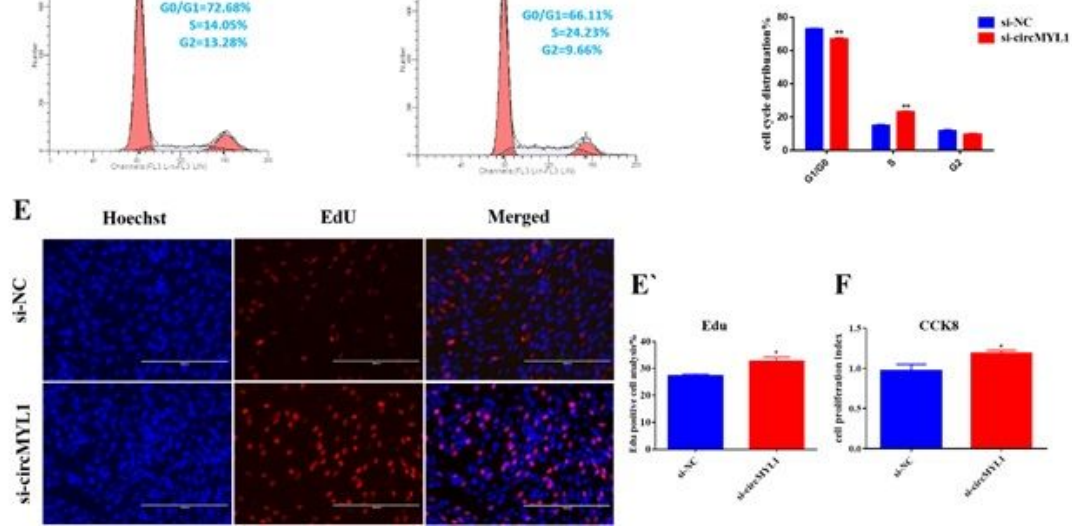

Figure 3

knockdown of circMYL1 promotes the proliferation of bovine primary myoblasts (A) qRT-PCR detected the interference effect of siRNA (si-circMYL1). (B) qRTPCR detected the relative expression levels of proliferation marker genes PCNA, CDK2, and CyclinD1. (C) western blot detected the protein expression levels of proliferation marker gene PCNA, CDK2, and CyclinD1. (C

) His $\rightarrow$ gramgraphshow $\in$ gtheprote $\in b$ and densityevaluatedusingIma $\geq J$. (D) Thecellcyc $\leq$ wasanalyzedbyflowcy $\rightarrow$ metry. ( $D$ ) Histogram graph showing the number of cells in each phase of the cell cycle. (E) The cell proliferation was detected by (EdU) assay Scale bar, $200 \mu m$. (E') Graph of a histogram showing the percentage of EdU-positive cells. (F) The cell counting kit-8 (CCK-8) assay was used to detect the cell proliferation index. Data are shown as mean \pm SEM. $N=3 .{ }^{*} \mathrm{P}<0.05 ; * * \mathrm{P}<0.01$. 
A

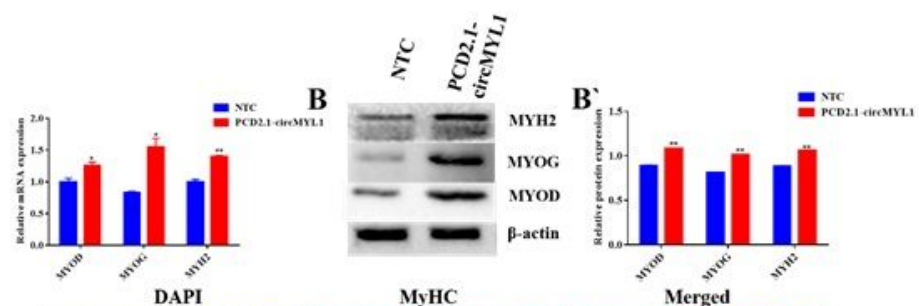

C
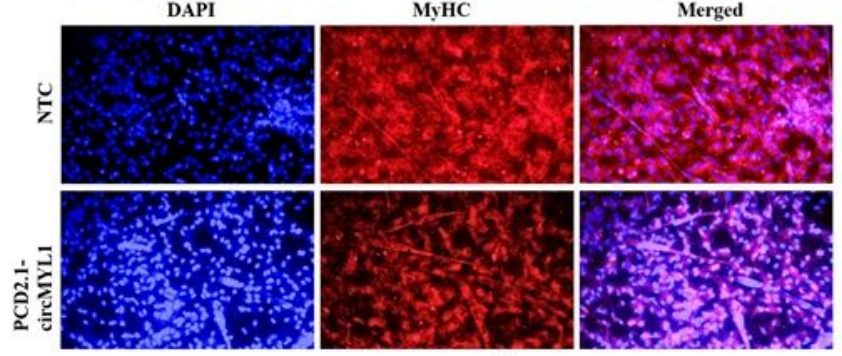

D

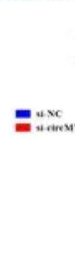

E

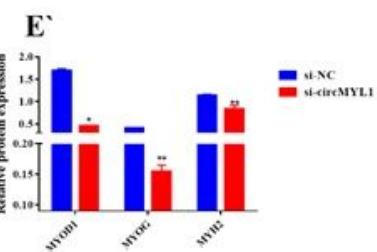

F
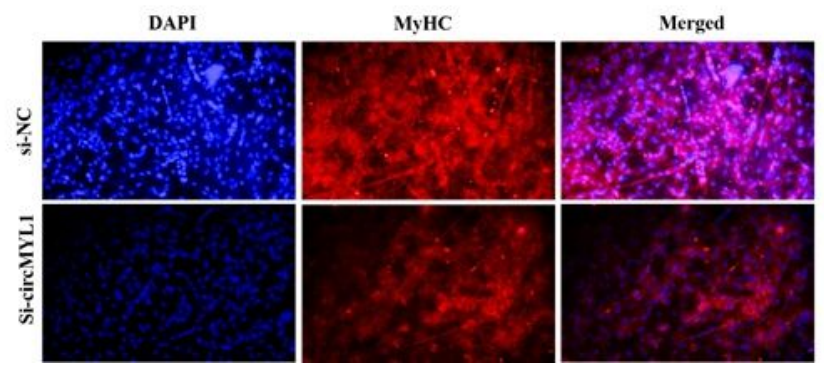

\section{Figure 4}

The effect of circMYL1 on the differentiation of myoblasts (A) qRT-PCR detected the relative expression levels of differentiation marker genes MyoD, MyoG, and MYH2 after transfected with circMYL1 overexpression vector. (B) Western blot detected the protein expression levels of differentiation marker genes MyoD, MyoG, and MYH2. (B

) His $\rightarrow$ gramgraphshow $\in$ gtheprote $\in b$ and densityevaluatedusingIma $\geq J$. (C) Im $\mu$ noflu or escencedetectedMyHC(red) - positivemyotub ) Histogram graph showing the protein band density evaluated using ImageJ. (F) Immunofluorescence detected MyHC (red)-positive myotubes after transfected by si-circMYL1. And they were detected under a fluorescence microscope: scale bar, $200 \mu \mathrm{m}$. Data are shown as mean $\pm \mathrm{SEM}$. N $=3$. ${ }^{\mathrm{P}}<0.05 ; * \star \mathrm{P}$ $<0.01$. 

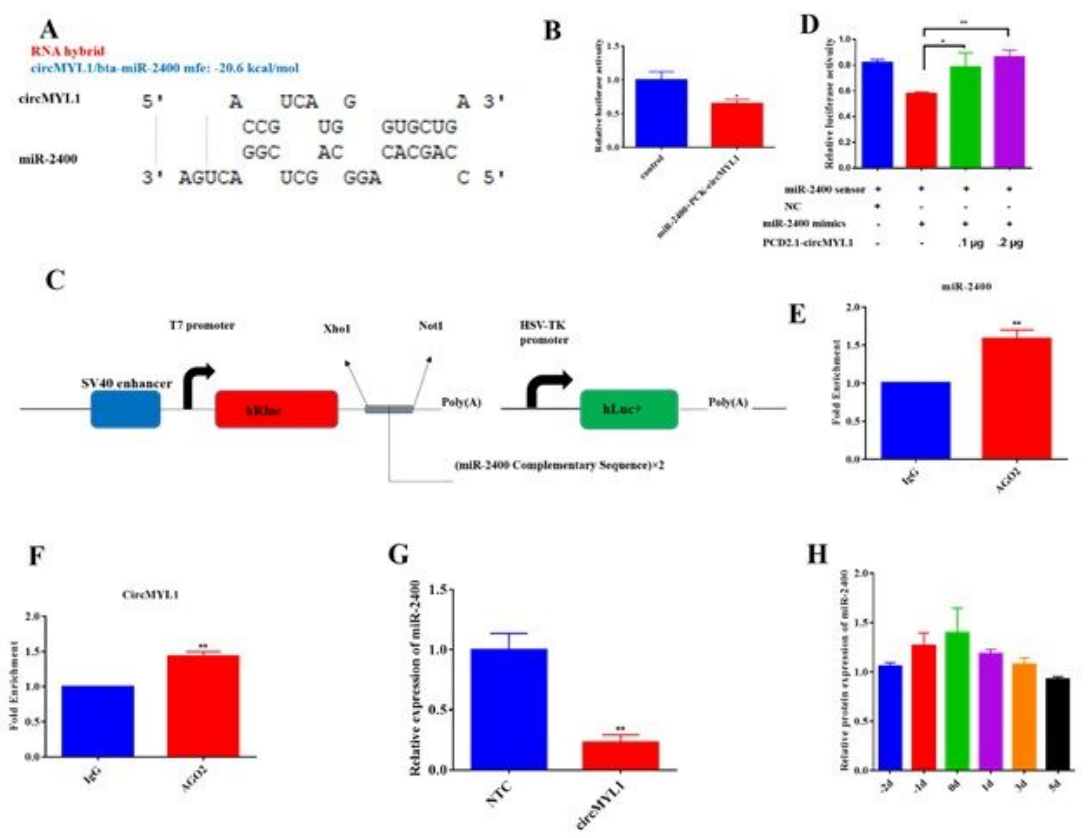

\section{Figure 5}

CircMYL1 acts as a miR-2400 sponge (A) RNAhybrid predicted the potential binding sites of miR-2400 on the circMYL1 sequence. (B) Luciferase assay was conducted after cotransfected the HEK293T cells with miR-2400 mimic and PCK-circMYL1. (C) A schematic drawing for the structure of the miR-2400 sensor. (D) Luciferase assay was conducted after cotransfected the miR-2400 sensor into HEK293 T cells with the miR-2400 mimic and/or PCD2.1-circMYL1. (E) AG02 RNA immunoprecipitation (RIP) assay detected the fold enrichment of miR-2400 in bovine primary myoblasts. (F) AGO2 RNA immunoprecipitation (RIP) assay detected the fold enrichment of circMYL1 in bovine primary myoblasts. (G) qRT-PCR detected the relative expression levels of miR-2400 after overexpression of the circMYL1. $(\mathrm{H})$ miR-2400 expression level during proliferation and differentiation of bovine primary myoblasts. Data are shown as mean \pm SEM. $N=3 .{ }^{*} P<0.05 ;{ }^{\star *} P<0.01$.
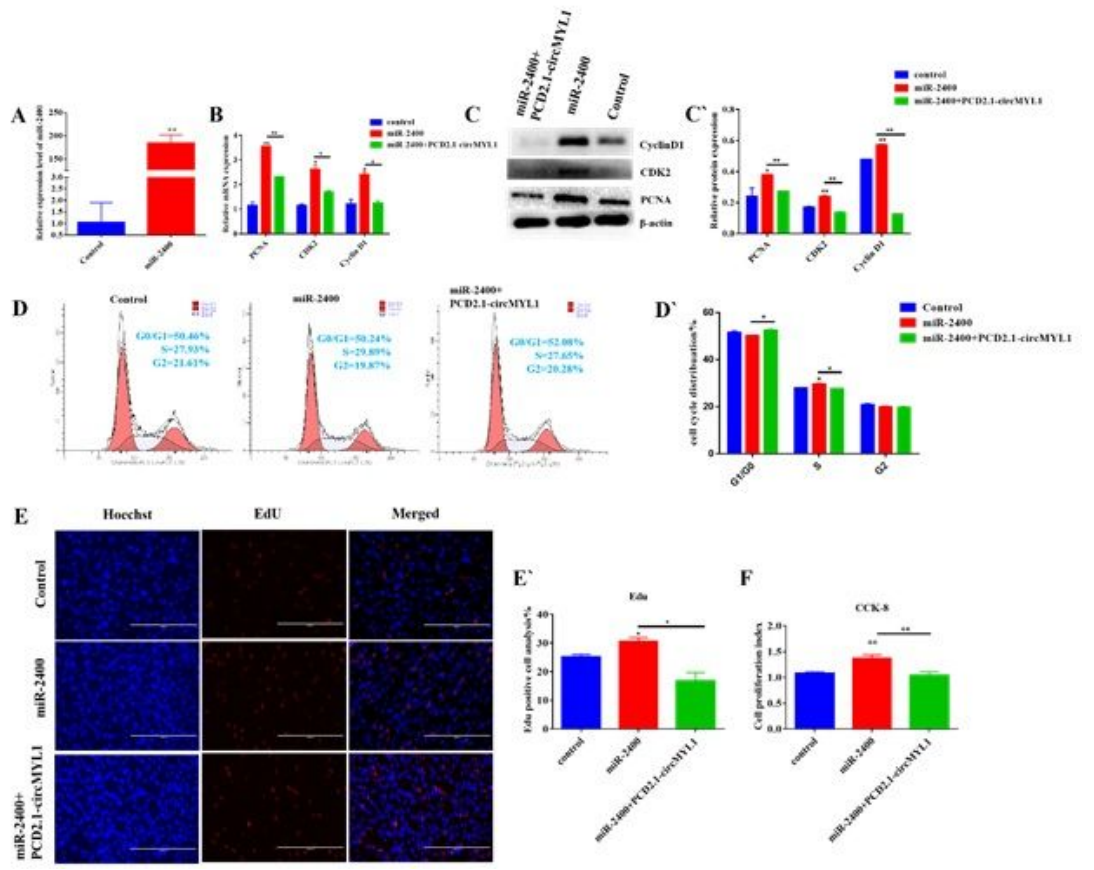

\section{Figure 6}

miR-2400 promotes myoblasts proliferation (A) qRT-PCR detected the transfection efficiency of miR-2400 mimic. (B) Bovine primary myoblasts were transfected with miR-2400 mimic and/or circMYL1 overexpression vector, and qRT-PCR detected the relative expression levels of proliferation marker genes PCNA, CDK2, and CyclinD1. (C) western blot detected the protein expression levels of proliferation marker gene PCNA, CDK2, and CyclinD1. (C ) His $\rightarrow$ gramgraphshow $\in$ gtheprote $\in b$ and densityevaluatedusingIma $\geq J$. (D) Thecellcyc $\leq$ wasanalyzedbyflowcy $\rightarrow$ metry. ( $D$ ) Histoaram araph showina the number of cells in each phase of the cell cycle. (E) The cell proliferation was detected by (EdU) assay Scale bar, $200 \mu \mathrm{m}$. (E') Loading [MathJax]/jax/output/CommonHTML/fonts/TeX/fontdata.js 
Graph of a histogram showing the percentage of EdU-positive cells. (F) The cell counting kit-8 (CCK-8) assay was used to detect the cell proliferation index. Data are shown as mean \pm SEM. $N=3 . * * P<0.05 ; * * P<0.01$.

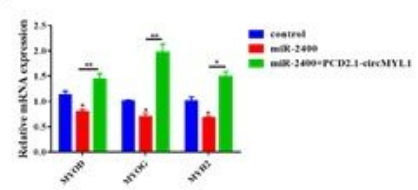

B

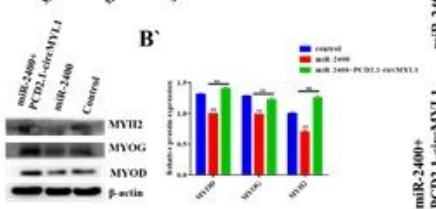

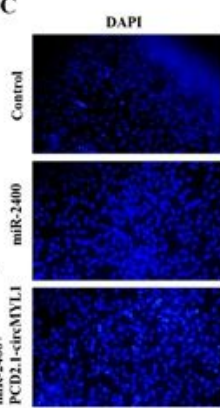
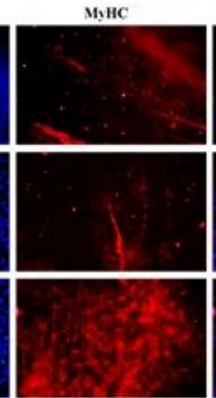

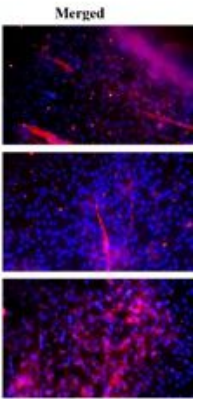

Figure 7

miR-2400 promotes myoblasts proliferation (A) qRT-PCR detected the transfection efficiency of miR-2400 mimic. (B) Bovine primary myoblasts were transfected with miR-2400 mimic and/or circMYL1 overexpression vector, and qRT-PCR detected the relative expression levels of proliferation marker genes PCNA, CDK2, and CyclinD1. (C) western blot detected the protein expression levels of proliferation marker gene PCNA, CDK2, and CyclinD1. (C ) His $\rightarrow$ gramgraphshow $\in$ gtheprote $\in b$ and densityevaluatedusingIma $\geq J$. (D) Thecellcyc $\leq$ wasanalyzedbyflowcy $\rightarrow$ metry. (D) Histogram graph showing the number of cells in each phase of the cell cycle. (E) The cell proliferation was detected by (EdU) assay Scale bar, $200 \mu \mathrm{m}$. (E') Graph of a histogram showing the percentage of EdU-positive cells. (F) The cell counting kit-8 (CCK-8) assay was used to detect the cell proliferation index. Data are shown as mean \pm SEM. $N=3 .{ }^{*} \mathrm{P}<0.05 ; * * \mathrm{P}<0.01$.

A

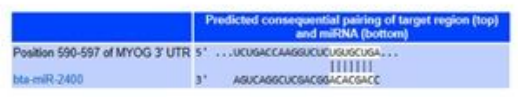

B

MYOG-3'UtR-WT 5 '...UCUGACCAAGguCUCUGUGCUGA... bta-miR-2400 3' AGUCAGGCUCGACGGACACGACC MYOG -3UTR-MUt 5 ....UCUGACCAAgGUCUCUGACGUGa

D

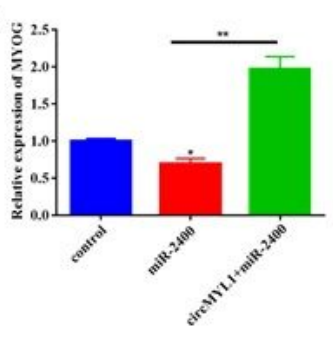

C

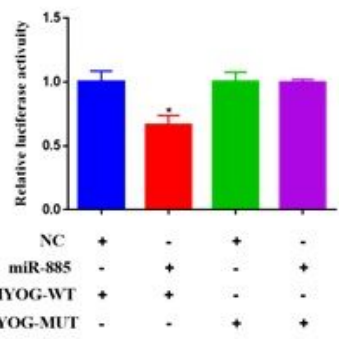

E

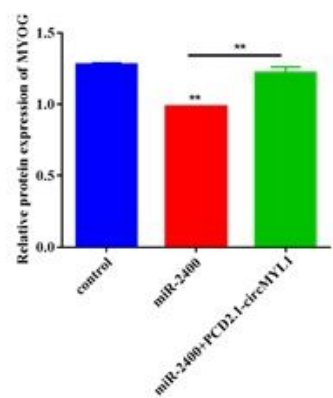

Figure 8

CircMYL1 acts as a ceRNA for miR-2400 to activate MYOG (A) TargetScan 7.2 predicted the potential binding site of miR-2400 in the MYOG-3'-UTR. (B) The sequence of miR-2400 and its predicted binding site in MYOG 3'-UTR and mutated 3'-UTR. (C) Luciferase assay was conducted after cotransfected the miR2400 mimic or negative control into HEK293 T cells with the MYOG-WT or MYOG-MUT. The activity of renilla luciferase was normalized for the activity of firefly luciferase. (D) qRT-PCR detected the relative expression levels of MYOG in bovine primary myoblasts after transfected with miR-2400 mimic and/or circMYL1 overexpression vector. (E) Western blot analysis detected the protein expression levels of MYOG in bovine primary myoblasts after transfected with miR-2400 mimic and/or circMYL1 overexpression vector. (E') Histogram graph showing the protein band density evaluated using ImageJ. Data are shown as mean \pm SEM. $N=3 . * P<0.05 ; * * P<0.01$. 


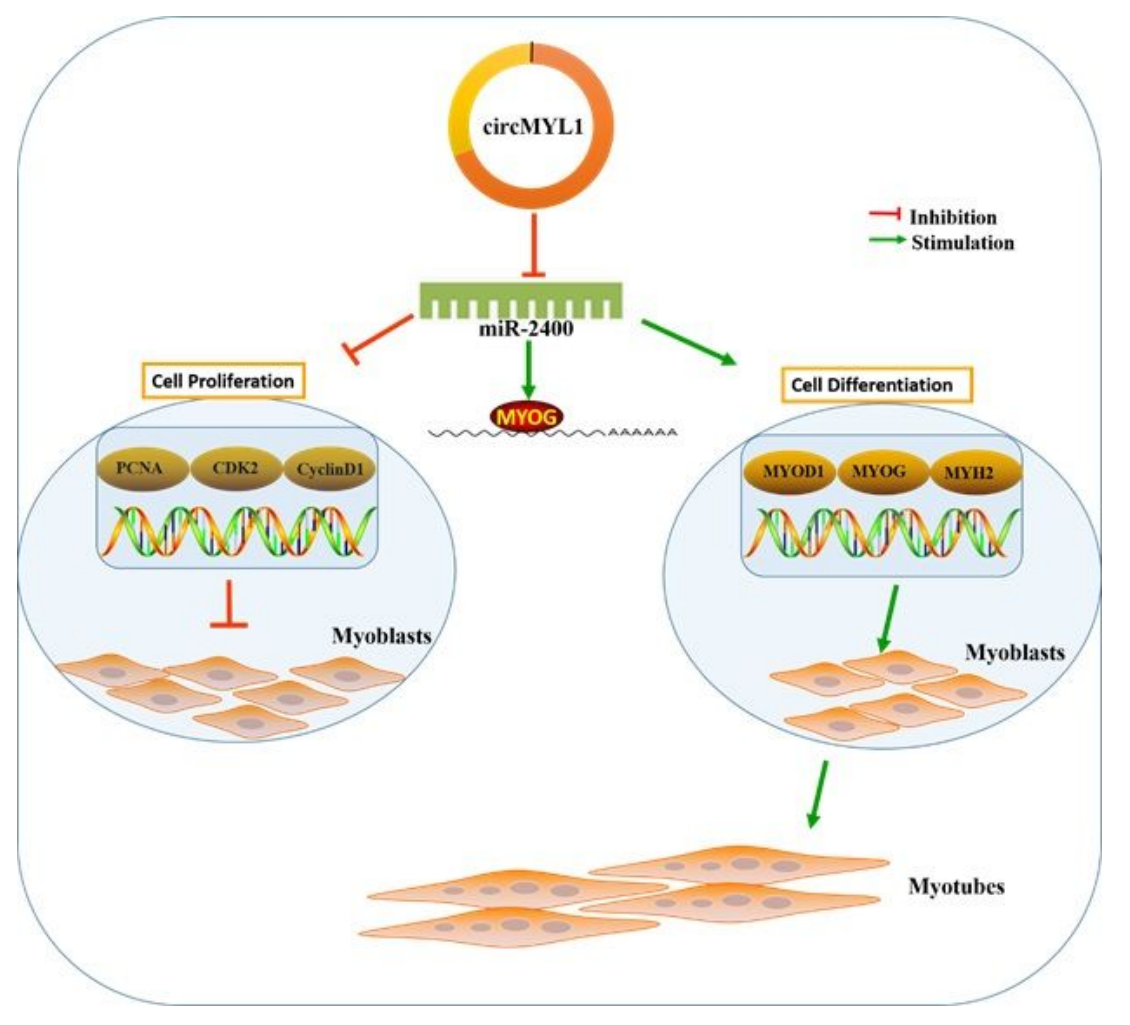

Figure 9

Schematic mechanism of circMYL1 in regulating bovine myogenesis. CircMYL1 regulates myogenesis by acting as a ceRNA for miR-2400 to activate MYOG. CircMYL1 inhibits the proliferation of bovine primary myoblasts through negative regulation of proliferation marker genes PCNA, CDK2, and CyclinD1. CircMYL1 promotes the differentiation of bovine primary myoblasts through the up-regulation of differentiation marker genes MyoD, MyoG, and MYH2.

\section{Supplementary Files}

This is a list of supplementary files associated with this preprint. Click to download.

- supplemantalinformation.docx 